\title{
Altered Functional Integration in the Salience and Default Mode Networks in Euthymic Pediatric Bipolar Disorder
}

\author{
Weifang Cao ${ }^{D},{ }^{1}$ Haoran Chen, ${ }^{2}$ Qing Jiao ${ }^{D},{ }^{1}$ Dong Cui, ${ }^{1}$ Yongxin Guo, ${ }^{1}$ Weijia Gao, ${ }^{3}$ \\ Jianfeng Qiu $\mathbb{D}^{1},{ }^{1}$ Linyan Su $\mathbb{D},{ }^{4}$ and Guangming $\mathbf{L u} \mathbb{D}^{5}$ \\ ${ }^{1}$ Department of Radiology, Shandong First Medical University \& Shandong Academy of Medical Sciences, Tai'an 271016, China \\ ${ }^{2}$ Department of Radiology, Shandong Cancer Hospital and Institute, Shandong First Medical University and Shandong Academy of \\ Medical Sciences, Jinan 250117, China \\ ${ }^{3}$ Department of Child Psychology, The Children's Hospital, Zhejiang University School of Medicine, Hangzhou 310003, China \\ ${ }^{4}$ Mental Health Institute of the Second Xiangya Hospital, Key Laboratory of Psychiatry and Mental Health of Hunan Province, \\ Central South University, Changsha 410011, China \\ ${ }^{5}$ Department of Medical Imaging, Jinling Hospital, Clinical School of Medical College, Nanjing University, Nanjing 210002, China
}

Correspondence should be addressed to Qing Jiao; bingbao17@163.com and Linyan Su; xysulinyan@126.com

Received 26 February 2020; Revised 28 August 2020; Accepted 21 September 2020; Published 9 October 2020

Academic Editor: F. Scott Hall

Copyright (C) 2020 Weifang Cao et al. This is an open access article distributed under the Creative Commons Attribution License, which permits unrestricted use, distribution, and reproduction in any medium, provided the original work is properly cited.

\begin{abstract}
Accumulating studies demonstrate emotional and cognitive dysregulation in the euthymic period of pediatric bipolar disorder (PBD). However, the relative contribution of functional integration in human brain to disturbed emotion and cognitive function in the euthymic PBD patients remains unclear. In this study, 16 euthymic PBD patients and 16 healthy controls underwent resting-state functional magnetic resonance imaging. A data-driven functional connectivity analysis was used to investigate functional connectivity changes of the euthymic PBD. Compared with healthy controls, the euthymic PBD exhibited greater global functional connectivity density in the left anterior insula and lower global functional connectivity density in the right temporoparietal junction, the left angular gyrus, and the bilateral occipital lobule. A distant functional connectivity analysis demonstrated altered integration within the salience and default mode networks in euthymic PBD. Correlation analysis found that altered functional connectivity of the salience network was related to the reduced performance in the backward digit span test, and altered functional connectivity of the default mode network was related to the Young Mania Rating Scale in euthymic PBD patients. Our findings indicated that disturbed functional integration in salience and default mode networks might shed light on the physiopathology associated with emotional and cognitive dysregulation in PBD.
\end{abstract}

\section{Introduction}

Bipolar disorder (BD) is a chronic and debilitating mental illness and has been increasingly diagnosed in pediatric age children (G. Z. [1]). In addition, retrospective studies indicated that symptoms in $55-60 \%$ of adults with BD begin in childhood or adolescence [2]. Therefore, it is important to understand the developmental pathophysiology of BD by investigating pediatric bipolar disorder (PBD). Substantial evidence indicates that cognitive impairment and emotional lability are present not only in periods of acute mood symptoms but also in periods of euthymia in PBD ([3]; G. Z. [1, 4]). However, little is known about the neurocognitive mechanisms of the euthymic phase of PBD.

Functional magnetic resonance imaging (fMRI) has been broadly used to investigate euthymic PBD [5, 6]. Many studies have provided evidence for changes of brain function in $\mathrm{BD}$, such as changes in the corticolimbic pathways during mood episode $[7,8]$. Recently, intrinsic functional connectivity (FC) based on resting-state fMRI has been employed to reveal stable and reliable functional brain networks, such as the default mode network (DMN), and the salience network (SN), which are associated with cognition and emotion $[9,10]$. Moreover, altered functional connectivity has been considered a potential 
biomarker for psychiatric disorders [11, 12]. Many imaging studies have demonstrated inconsistent functional changes in brain networks in different neural phenotypes in BD patients due to heterogeneity in the analysis methods used $[5,13]$. However, intrinsic functional connectivity analysis provides a useful approach to investigate the neural architecture of euthymic PBD patients.

In the current study, we firstly evaluated the global functional connectivity feature by using an unbiased data-driven method. These features are obtained from global functional connectivity density (FCD), which may be more sensitive in detection of functional alterations of the distribution of brain hubs [14, 15]. Then, we assessed the distant functional connectivity of those brain regions in which we observed a significantly different global FCD in euthymic PBD patients. We predicted that the euthymic PBD patients would exhibit altered functional connectivity, and these changes may contribute to the neural physiopathology of PBD.

\section{Methods}

2.1. Participants. This study recruited 16 PBD patients during clinical remission from the child and adolescent psychiatric clinic of the Second Xiangya Hospital of Central South University (Changsha, P. R. China) from January to July, 2012. All patients were required to meet the Diagnostic and Statistical Manual for Mental Disorders, Fourth Edition (DSM-IV) criteria for BD with a current remission episode. The exclusion criteria included the diagnosis of bipolar disorder subtype or current mixed episode. Sixteen age- and sex-matched healthy control (HC) subjects were recruited through advertisements in local public schools. All participants were 10-17 years old, right-handed and of Han ethnicity. They could follow the instructions to keep still during MRI scanning. The additional exclusion criteria for all participants included the presence of major sensorimotor handicaps, history of neurological disorder, history of electroconvulsive therapy, lower score of full-scale intelligence quotient (IQ < 80), and standard fMRI contraindications (e.g., metallic implants, retractors or braces, and claustrophobia). The study was approved by the ethics committee of the Second Xiangya Hospital of Central South University. Written informed consent was obtained from the parents of all participants.

2.2. Clinical and Psychological Assessment. All patients were diagnosed independently by two experienced psychiatrists for Affective Disorders and Schizophrenia for School aged Children Present and Lifetime Versions (K-SADS-PL). Current mental states were assessed using the Young Mania Rating Scale (YMRS) and Mood and Feelings Questionnaire (MFQ). To evaluate the effects of PBD on cognitive function, psychological measurements for all participants included the Digit Span Test (DST), the Trail Marking Test (TMT), and the Stroop Color-Word Test (SCWT). The DST includes the forward digit span test (DST-F) and backward digit span test (DST-B) to evaluate attention and executive processes. The SCWT is used to assess the ability to inhibit cognitive interference and is composed of three parts. The participant is asked to name the color of a series of dots in SCWT-A, to name the color-ink words in SCWT-B, and to name the color of words whose meanings are different from ink colors in SCWT-C. The TMT is frequently used neuropsychological tests in clinical practice and is comprised of part A (TMT-A) and part B (TMT-B). The TMT-A includes numbered circles only, whereas the TMT-B contains more numbered circles and squares. The participant is asked to connect these numbers in order.

2.3. Image Acquisition and Preprocessing Analysis. All image data were acquired using a Siemens 3T Siemens Trio scanner (Siemens, Munich, Germany). To minimize head motion, we fixed the subjects' heads using foam pads. The axial T1weighted anatomical images were acquired using a spoiled gradient recall sequence, generating 176 slices (repetition time $(\mathrm{TR})=2300 \mathrm{~ms}$, echo time $(\mathrm{TE})=2.03 \mathrm{~ms}$, flip angle $(\mathrm{FA})=$ $9^{\circ}$, slice thickness $=1 \mathrm{~mm}$, matrix size $=256 \times 256$, and field of view $(\mathrm{FOV})=256 \mathrm{~mm} \times 256 \mathrm{~mm})$. Resting-state functional images were collected using echo planar imaging (EPI) $\left[\mathrm{TR}=2000 \mathrm{~ms}, \mathrm{TE}=30 \mathrm{~ms}, \mathrm{FA}=90^{\circ}, \mathrm{FOV}=240 \mathrm{~mm} \times 240\right.$ $\mathrm{mm}$, matrix $=64 \times 64$, and slice thickness $=4 \mathrm{~mm}]$, generating 30 slices. The functional scanning lasted for $510 \mathrm{~s}$, yielding a total of 255 volumes. During the resting-state scanning, the subjects were asked to lie with their eyes closed, not to fall asleep, and not to think of anything in particular. To ensure magnetic field stabilization, the first five volumes were discarded.

All image data were preprocessed using the Neuroscience Information Toolbox (NIT) [16]. The preprocessing procedure included slice time correction, realignment, and spatial normalization $\left(3 \times 3 \times 3 \mathrm{~mm}^{3}\right)$. We excluded subjects with exceeded head motion (more than $2 \mathrm{~mm}$ translation or $2^{\circ}$ rotational movements). The linear regression analysis was utilized to remove several nuisance covariates from the time course of all brain voxels of the functional data. These covariates included 12 head motion parameters (six head parameters and its derivative), white matter signal, cerebrospinal fluid signal, and global signal. Then, the temporal passband filtering $(0.01-0.08 \mathrm{~Hz})$ was conducted on the time courses of functional data to remove low-frequency drift and to minimize high-frequency physiological noise.

2.4. Global Functional Connectivity Density Analysis. For each subject, the global FCD map was obtained using Pearson's linear correlation from individual preprocessed time course. The threshold of the correlation coefficient, Tc, was used to determine significant connection between two voxels. The global FCD value at a given voxel was defined as the number of voxels with significant connections in the whole brain with the given voxel. The global FCD map reflected the total number of functional connections per voxel.

We chose a dynamic threshold range from 0.4 to 0.8 in 0.05 steps, to obtain more reliable and robust findings. The total nine Tc thresholds were used in our study. To address variability in all global FCD across subjects, each individual global FCD map was normalized by dividing by the mean value across voxels in a given subject. For all FCD maps, we created nine normalized maps for the nine Tc thresholds for each subject. Spatial smooth with $6 \mathrm{~mm}$ Gaussian kernel 
TABLE 1: Comparison of clinical and neuropsychological measurement between the euthymic PBD patients and healthy controls.

\begin{tabular}{|c|c|c|c|}
\hline & Euthymic PBD & $\mathrm{HC}$ & $P$ value \\
\hline Gender (male/female) & $7 / 9$ & $5 / 11$ & $0.716^{\mathrm{a}}$ \\
\hline Age (mean $\pm S D$, years) & $15.12 \pm 1.71$ & $14.06 \pm 1.48$ & $0.070^{\mathrm{b}}$ \\
\hline Education (mean $\pm \mathrm{SD}$, years) & $8.19 \pm 1.80$ & $7.19 \pm 2.04$ & $0.152^{\mathrm{b}}$ \\
\hline Age of onset (mean $\pm \mathrm{SD}$, years) & $13.13 \pm 2.09$ & & \\
\hline BD type (I/II) & $10 / 6$ & & \\
\hline Familial history (yes/no) & $5 / 11$ & & \\
\hline \multicolumn{4}{|l|}{ Medications } \\
\hline Lithium & $6(38 \%)$ & & \\
\hline Valproate & $11(69 \%)$ & & \\
\hline Atypical antipsychotics & $13(81 \%)$ & & \\
\hline \multicolumn{4}{|l|}{ K-SADS-PL comorbidity diagnoses } \\
\hline $\mathrm{ADHD}$ & $4(25 \%)$ & & \\
\hline OCD & $1(6 \%)$ & & \\
\hline Anxiety & $3(19 \%)$ & & \\
\hline Tic & $1(6 \%)$ & & \\
\hline MFQ score $($ mean \pm SD) & $7.50 \pm 4.37$ & $5.56 \pm 3.18$ & $0.385^{\mathrm{c}}$ \\
\hline IQ $($ mean $\pm S D)$ & $106.69 \pm 10.54$ & $105.00 \pm 7.00$ & $0.609^{c}$ \\
\hline YMRS score $($ mean $\pm S D)$ & $5.38 \pm 1.69$ & $3.59 \pm 1.90$ & $0.009^{c}$ \\
\hline SCWT-A $($ mean \pm SD $)$ & $55.94 \pm 11.34$ & $64.81 \pm 11.34$ & $0.003^{\mathrm{c}}$ \\
\hline SCWT-B $($ mean \pm SD) & $73.88 \pm 13.41$ & $86.56 \pm 8.71$ & $0.001^{\mathrm{c}}$ \\
\hline SCWT-C $($ mean \pm SD $)$ & $33.63 \pm 7.54$ & $39.50 \pm 7.84$ & $0.006^{\mathrm{c}}$ \\
\hline TMT-A $($ mean \pm SD) & $37.83 \pm 13.48$ & $30.50 \pm 9.29$ & $0.004^{\mathrm{c}}$ \\
\hline TMT-B $($ mean \pm SD $)$ & $98.44 \pm 49.70$ & $82.17 \pm 29.51$ & $0.237^{\mathrm{c}}$ \\
\hline DST-F (mean \pm SD) & $8.75 \pm 1.81$ & $9.00 \pm 1.10$ & $0.758^{\mathrm{c}}$ \\
\hline DST-B $($ mean \pm SD $)$ & $4.50 \pm 1.41$ & $5.87 \pm 1.63$ & $0.019^{c}$ \\
\hline
\end{tabular}

${ }^{\mathrm{a}}$ Chi-square test; ${ }^{\mathrm{b}}$ two-sample $t$-test; ${ }^{\mathrm{c}}$ two-sample $t$-test controlled by age, gender, and education years. ADHD: attention-deficit/hyperactivity disorder; DST-B: backward digit span test; DST-F: forward digit span test; HC: healthy controls; IQ: intelligence quotient; K-SASADS-PL: Schedule for Affective Disorders and Schizophrenia for School-Age Children-Present and Lifetime Version; MFQ: Child Mood and Feelings Questionnaire; OCD: obsessive-compulsive disorder; PBD: pediatric bipolar disorder; SCWT: Stroop Color-Word Test; TMT-A: part A of trail making test; TMT-B: part B of trail making test; YMRS: Young Mania Rating Scale.

of full-width half maximum (FWHM) was used to minimize the differences in the functional anatomy of the brain across subjects.

Group analyses of all global FCD maps were conducted using one-sample $t$-tests $(P<0.05$, FDR corrected). For each global FCD map for each threshold, Tc, we conducted a twosample $t$-test comparing the euthymic PBD and HC groups, controlling for the age, gender, and education years.

To identify robust differences of global FCD between the euthymic PBD and HC groups, we only included the clusters exhibiting a significant difference in at least 4 consecutive Tc values of FCD comparisons. Then, these clusters were used as regions of interest (ROIs) for the subsequent functional connectivity analyses.

2.5. Functional Connectivity Analysis. For the FC analysis, the normalized functional images were further processed using spatial smoothing using a $6 \mathrm{~mm}$ Gaussian kernel of FWHM. Then, the general regression analysis was performed to minimize the reflection of nuisance signals, as mentioned above. After filtering (0.01-0.08 Hz), FC analysis was performed by calculating the Pearson's correlation coefficients between the average time course of each ROI and that of each voxel in the whole brain. A Fisher $z$-transformation for correlation coefficients was applied. Therefore, individual $Z$-score FC maps were defined for each ROI and subject. One-sample $t$-tests were conducted within the euthymic PBD and HC groups. Then, we performed two-sample $t$ -tests to detect the differences in the FC maps between the euthymic PBD and $\mathrm{HC}$ groups within the masks from the union set of the one-sample tests results of the FC maps ( $P<0.05$, FDR corrected) of the two groups, controlling for age, education years, and gender effects.

2.6. Correlation Analyses between Functional Connectivity and Clinical and Psychological Measurements. We used a partial correlation analysis to explore the relationship between clinical and psychological measurements and FC features including the global FCD of each ROI and the FC map of ROIs, controlling for the effects of age, education years, and gender. 


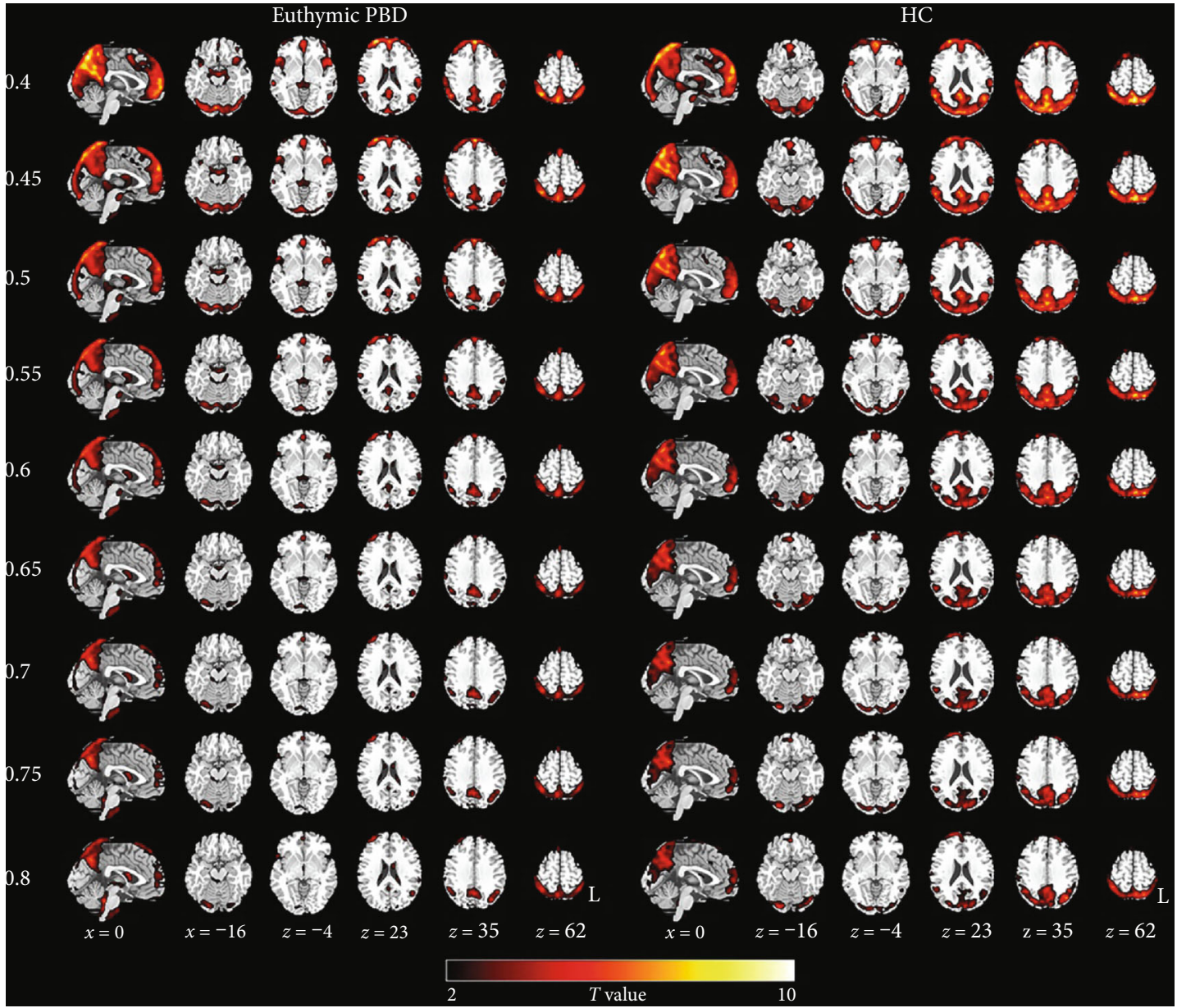

FIgUre 1: The global FCD maps for the nine Tc thresholds in the euthymic pediatric bipolar disorder (PBD) and healthy controls (HC) groups (one-sample $t$-test, $P<0.05$, FDR corrected, cluster size $>621 \mathrm{~mm}^{3}$ ). L: left; R: right.

\section{Results}

There was no significant difference in gender, age, education years, IQ, MFQ, TMT, and DST-F scores between the euthymic PBD and HC groups $(P>0.05)$. The YMRS, SCWT, and DST-B scores showed significant difference between the two groups $(P<0.05)$. Table 1 shows these clinical data and neuropsychological measurement in detail.

3.1. Global Functional Connectivity Density Analysis. We illustrated the distribution of the global FCD for the nine Tc in the euthymic PBD and HC groups (Figure 1). For almost all Tc thresholds, we found the highest global FCD in the precuneus, angular gyrus, inferior parietal lobule, occipital cortex, superior temporal cortex, superior frontal gyrus, and the cerebellum, while the cluster size of the regions grew smaller as the Tc threshold increased. The pattern of regions exhibiting highest global FCD was similar as that of previous studies.

We conducted a two-sample $t$-test to compare data from the euthymic PBD group and HC group for each threshold $\left(P<0.005\right.$, cluster size $\left.>270 \mathrm{~mm}^{3}\right)$. We summarized all differences of global FCD resulting from the nine Tc thresholds to obtain stable differences between groups (Figure 2).

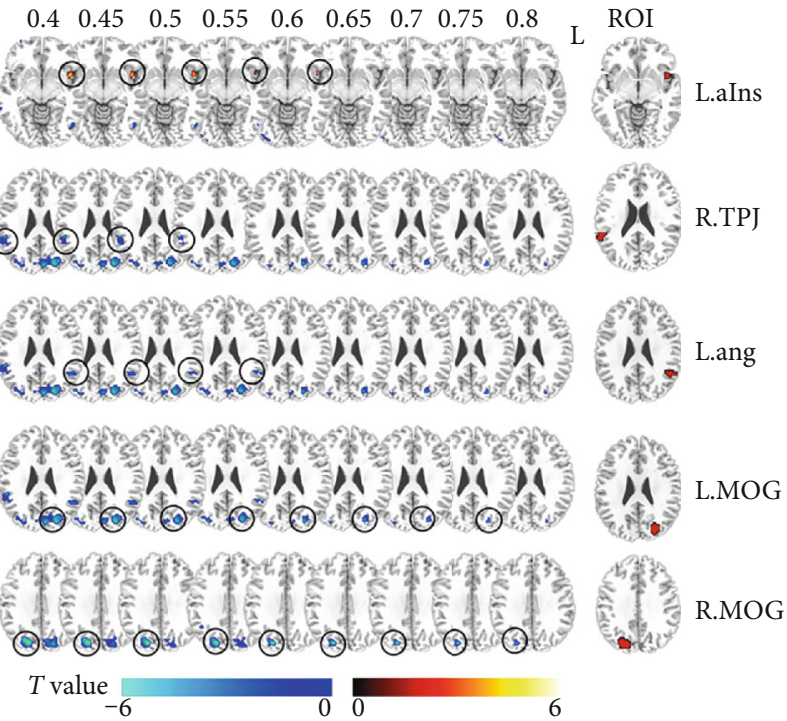

Figure 2: The differences of global FCD maps between the euthymic PBD and HC groups in 9 Tc thresholds separately $(P<0.005$, cluster size $\left.>270 \mathrm{~mm}^{3}\right)$. The left part shows 5 ROIs' position and designation; L: left. ROIs' abbreviations are consistent with those shown in Table 2. 
TABLE 2: Five ROIs determined in global FCD analysis.

\begin{tabular}{lcccccc}
\hline \multirow{2}{*}{ Brain regions } & \multirow{2}{*}{ Abbreviation } & \multicolumn{2}{c}{ Peak MNI coordinate } & \multirow{2}{*}{ Brodmann } & \multirow{2}{*}{ Size of ROIs $\left(\mathrm{mm}^{3}\right)$} \\
\hline Left anterior insula & L.aIns & -45 & 9 & -9 & 48 & 459 \\
Right temporoparietal junction & R.TPJ & 51 & -39 & 24 & 42 & 405 \\
Left angular & L.Ang & -45 & -51 & 21 & 39 & 918 \\
Left middle occipital gyrus & L.MOG & -24 & -84 & 21 & 18,19 & 3051 \\
Right middle occipital gyrus & R.MOG & 21 & -81 & 33 & 18,19 & 324 \\
\hline
\end{tabular}

FCD: functional connectivity density; MNI: Montreal Neurological Institute; ROI: regions of interest.

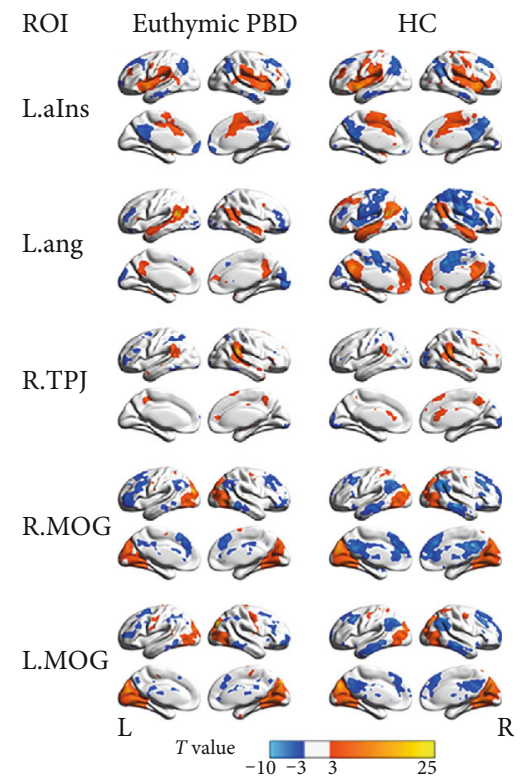

FIGURE 3: The group-level functional connectivity maps seeded at 5 ROIs in the euthymic pediatric bipolar disorder (PBD) and healthy control (HC) groups (one-sample $t$-test, $P<0.05$, FDR corrected, cluster size $>621 \mathrm{~mm}^{3}$ ). L: left; R: right. ROIs' abbreviations are consistent with those shown in Table 2.

Compared with the HC group, the euthymic PBD group showed increased global FCD in the left anterior insula at five consecutive Tc thresholds comparisons (from 0.4 to 0.6 ). We also found significantly decreased global FCD for more than half of thresholds at four clusters in the euthymic PBD group compared with the HC group. These clusters included the right angular gyrus, the left temporoparietal junction (TPJ), and the bilateral middle occipital cortex (MOG). In total, we identified five clusters of significantly altered global FCD in the euthymic PBD group. These regions were defined as regions of interest (ROIs) in the subsequent FC analysis (Table 2).

3.2. Functional Connectivity Analysis. For each ROI, we assessed a whole brain FC map for each group using one-sample t-test $\left(P<0.05\right.$, FDR-corrected, cluster size $\left.>621 \mathrm{~mm}^{3}\right)$. Figure 3 shows these connectivity patterns of the FC map in the euthymic PBD and HC groups. In both groups, the regions that showed a positive correlation with the left anterior insula included the bilateral insula, dorsal anterior cingulate cortex, bilateral TPJ, supplementary motor area, and bilateral middle prefrontal cortex, which had been identified as the salience network in previous studies. The right supramarginal gyrus showed positive correlation with bilateral supramarginal cortex, the right middle prefrontal cortex, the anterior cingulate cortex, and precuneus. The left angular, which was identified as a node of the default mode network, positively correlated with the precuneus, posterior cingulate cortex, bilateral ventromedial prefrontal and inferior parietal cortex, and bilateral superior temporal gyrus and crus II of the cerebellum. In two groups, the seeds of bilateral MOG positively correlated with the occipital cortex, cuneus lobe, calcarine gyrus, and lingual gyrus.

The results of the two-sample $t$-test showed that four of the five ROIs exhibited significant differences between the euthymic $\mathrm{PBD}$ and $\mathrm{HC}$ groups $(P<0.005$, cluster size $>621$ $\mathrm{mm}^{3}$ ) (Figure 4). Compared with the $\mathrm{HC}$, the euthymic PBD exhibited decreased FC between the left anterior insula and right anterior insula and increased FC between the left anterior insula and the right TPJ. In the FC map of the left angular as seed, the euthymic PBD exhibited increased FC in the temporal gyrus and decreased FC in the bilateral angular and precuneus. The decreased FC between the bilateral MOG and the middle-inferior occipital cortex and cuneus were also identified in the euthymic PBD group (Table 3).

3.3. Results of Correlation Analyses. The correlation analysis showed a negative relationship in the FC between the left anterior insula and the left TPJ and the DST-B $(r=-0.559$, $P=0.024)$ in the euthymic PBD group. The functional connectivity between the left angular and the right angular was negatively correlated to the YMRS score $(r=-0.57, P=$ 0.022 ) in the euthymic PBD group (Figure 5).

\section{Discussion}

In this study, we demonstrated altered intrinsic FC in the brain of euthymic PBD using resting-state fMRI. We observed increased global FCD in the left anterior insula and decreased global FCD in the right TPJ, the left angular and bilateral occipital lobule in the euthymic PBD patients. Functional connectivity analyses based on ROI showed a distinct altered integration within the salience and default mode networks in euthymic PBD. Our findings indicate that resting-state FC based on a data-driven approach could be useful for evaluating altered pathophysiology in euthymic PBD patients. 


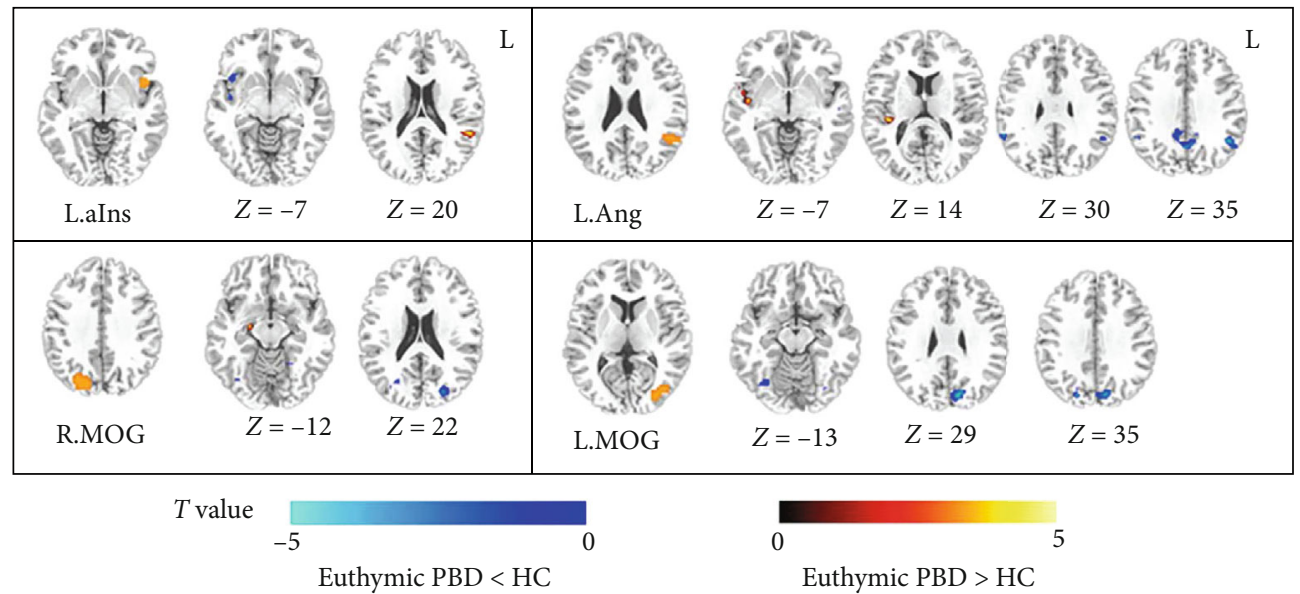

FIGURE 4: The difference of functional connectivity maps seeded at 4 ROIs between the euthymic pediatric bipolar disorder (PBD) and healthy control (HC) groups $\left(P<0.005\right.$, cluster size $\left.>621 \mathrm{~mm}^{3}\right)$. The left column for each subgraph shows the seed. The hot color represents higher correlation coefficients, and the cold color represents lower correlation coefficients in the euthymic pediatric bipolar disorder (PBD). ROIs' abbreviations are consistent with those shown in Table 2.

TABLE 3: The significantly altered functional connectivity between the euthymic PBD and HC groups in 4 seed maps.

\begin{tabular}{|c|c|c|c|c|c|c|}
\hline \multirow{2}{*}{ ROI } & \multirow{2}{*}{ Regions } & \multicolumn{3}{|c|}{ MNI coordinate } & \multirow{2}{*}{$T$ value } & \multirow{2}{*}{ Cluster size $\left(\mathrm{mm}^{3}\right)$} \\
\hline & & $x$ & $y$ & $z$ & & \\
\hline \multirow{2}{*}{ L.aIns } & Right insula & 42 & 15 & -12 & -3.51 & 648 \\
\hline & Left temporoparietal junction & -48 & -45 & 1 & 4.61 & 1080 \\
\hline \multirow{4}{*}{ L.Ang } & Right superior temporal cortex & 37 & -30 & 14 & 5.62 & 1674 \\
\hline & Left angular & -51 & -54 & 33 & -4.37 & 1215 \\
\hline & Right angular & 56 & -51 & 26 & -4.15 & 702 \\
\hline & Precuneus & 3 & -59 & 33 & -4.17 & 1566 \\
\hline \multirow{2}{*}{ L.MOG } & Left cuneus & -9 & -84 & 24 & -4.78 & 3078 \\
\hline & Right inferior occipital cortex & 39 & -75 & -9 & -3.99 & 1971 \\
\hline \multirow{2}{*}{ R.MOG } & Right hippocampus & 24 & -12 & -15 & 5.57 & 648 \\
\hline & Left cuneus & -24 & -84 & 21 & -4.78 & 3024 \\
\hline
\end{tabular}

ROIs' abbreviations are consistent with those shown in Table 2. HC: healthy controls; MNI: Montreal Neurological Institute; PBD: pediatric bipolar disorder; ROI: regions of interest.

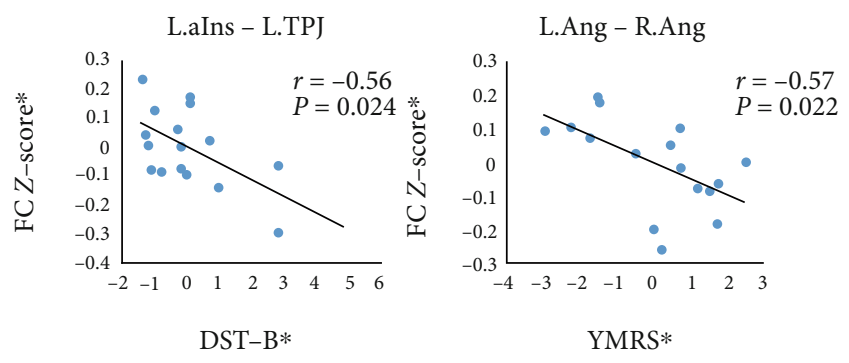

FIGURE 5: The relationship between the functional connectivity and psychological and clinical variables. * represents the adjusted values controlling for the influence of the age, gender, and education years by the linear regression model.

Using global FCD analysis, we observed significantly increased global functional connectivity in the left insula and decreased global functional connectivity in the right TPJ in the euthymic PBD patients compared to controls. The anterior insula and TPJ have been identified as key regions involved in the salience network, which is implicated in monitoring, detecting, and regulating salient stimuli from the internal and external environment [17]. This integration of the salience network may play a role for fundamental cognitive and behavioral function, and its alteration was 
found to associate with alteration of psychiatric symptoms in psychosis [18, 19]. Consistent with previous researches, abnormal emotional processing, as well as cognitive impairment, such as attention, processing speed, and working memory, characterized euthymic PBD [4, 20]. In the study, we observed impaired neuropsychological functioning, including decreased scores of the CWSW and DST-B and more completion time of TMT-A in euthymic PBD patients compared to controls. These measurements are commonly employed to examine cognitive function, and successful completion of these tests requires reasonable allocation of attention, flexibility, and working memory [21, 22]. The insula is considered a critical hub in mediating dynamic interactions in oriented attention and internally self-related cognition [23]. The TPJ, which is located at the ventralanterior section of the inferior parietal lobule and the posterior end of the superior temporal sulcus, is thought to either shift attention to goal-directed cognitive processing or understand others' mental state [24-26]. A recent task fMRI study had suggested that BD patients showed less activation and altered FC in regions within the salience network during an emotion-cognition integration task [7]. Our further voxelbased whole FC analysis showed enhanced FC between the left insula and the TPJ and decreased FC between the bilateral insula in the euthymic PBD patients. We found a negative correlation between the DST-B and FC between the left insula and the left TPJ in the euthymic PBD. Considering the critical role of the insula and TPJ in cognitive and emotional processes, our finding suggested that the altered functional integration within the salience network in euthymic PBD might contribute to the underlying mechanisms of impaired higher-level cognitive processes in patients.

Growing evidence has suggested that the DMN is compromised in many neuropsychiatric disorders $[6,27,28]$. The DMN, which contains the ventral-middle prefrontal cortex, bilateral angular gyrus, the precuneus (poster cingulate cortex), and bilateral temporal gyrus, is associated with self-referential and introspective states [29]. The left angular gyrus has been repeatedly implicated in episodic and semantic cognition, and the connection of the angular gyri with other DMN regions may act as a connector hub for global integration of information [30]. We observed the increased global FCD in the left angular gyrus in euthymic PBD patients. Further FC analysis revealed that the left angular gyrus showed increased FC with the right superior temporal cortex and decreased FC with the precuneus and right angular gyrus. Here, we observed a negative correlation between the FC of bilateral angular gyrus and the YMRS in the euthymic PBD. In other words, the lower FC between the bilateral angular gyrus was associated with the worse mental state. The precuneus involvement in introspective processes such as self-referential and emotional processing was associated with attenuated activity toward external events [29]. Therefore, we suggested that the altered interaction of regions of the DMN might serve to impair mental processing in the euthymic PBD patients.

The euthymic PBD patients exhibited decreased global FCD in the bilateral MOG, which may serve as the potential functional basis for the deficits in visual processing [31]. A few task fMRI studies found abnormalities in MOG during emotion face processing task in PBD patients $[32,33]$. Our previous study also found decreased functional activity in MOG, which was associated with the performance of cognition in PBD patients (W. [34]). Therefore, we speculated that the reduced FC in the MOG might impair the perception during self-referential processing in the euthymic PBD patients.

Several limitations of our study must be noted. First, the sample size of participants was relatively small, and the larger samples would help to confirm results presented here. Second, our sample included the euthymic PBD patients with type I and type II. Although we agree that there might be differences in FC in subtypes of PBD, it would have been very difficult to exclude an entire subtype of $\mathrm{BD}$ according to our study design. Finally, the majority of PBD patients were taking psychotropic medication when scanning. Few studies have suggested that drug treatment may affect functional neuroimaging measures [35]. Future studies will use the prospective design to clarify the contribution of medicine and subtypes of PBD to altered neuroimaging features of PBD patients.

\section{Conclusions}

In summary, we demonstrated that the euthymic PBD exhibited increased global FCD in the left anterior insula and decreased global FCD in the left angular and bilateral occipital lobule. Functional connectivity analyses showed a distinct altered integration within the salience and default mode networks in euthymic PBD. The altered functional integration was associated with altered emotional and cognitive processing in the euthymic PBD. These findings indicate that disturbed functional integration in salience and default mode networks might have potential implications for the physiopathology of the PBD.

\section{Data Availability}

The fMRI data used to support the findings of this study are available from the corresponding author upon request.

\section{Conflicts of Interest}

The authors declare that they have no conflict of interests.

\section{Authors' Contributions}

Author contributions included conception and study design (Weifang Cao, Qing Jiao, and Guangming Lu), data collection or acquisition (Weijia Gao, Yan Yin, and Linyan Su), statistical analysis (Dong Cui, Xiaojuan Wang, and Haoran Chen), interpretation of results (Weifang Cao, Yongxin Guo, and Jianfeng Qiu), drafting the manuscript work or revising it critically for important intellectual content (Weifang Cao, Qing Jiao, and Haoran Chen), and approval of the final version to be published and agreement to be accountable for the integrity and accuracy of all aspects of the work (all authors). 


\section{Acknowledgments}

We are grateful to all the participants in this study. This work was supported by the Funds for the National Natural Science Foundation of China (81371531 to Qing Jiao; 81901730 to Weifang Cao), Science and Technology Project of the Education Department of Shandong Province (J18KA262 to Weifang Cao), High-Level Cultivation Program of Shandong First Medical University and Shandong Academy of Medical Sciences (2017GCC11 to Weifang Cao), Taishan Scholars Program of Shandong Province (TS201712065 to Jianfeng Qiu), and Key Project of Scientific Research of "12th FiveYear Plan" in the Medical Research of the Army (BWS11J063 to GL).

\section{References}

[1] G. Z. Gao, Q. C. Jiao, Y. L. Ding, and L. Chen, "Study on quantitative assay of chondroitin sulfate with a spectrophotometric method of azure a," Guang Pu Xue Yu Guang Pu Fen Xi, vol. 23, no. 3, pp. 600-602, 2003.

[2] Q. Jiao and Q. Liu, "Simple spectrophotometric method for the estimation of algal polysaccharide concentrations," Journal of Agricultural and Food Chemistry, vol. 47, no. 3, pp. 996-998, 1999.

[3] L. R. Elias, K. W. Miskowiak, A. M. O. Vale et al., "Cognitive impairment in euthymic pediatric bipolar disorder: a systematic review and meta-analysis," Journal of the American Academy of Child and Adolescent Psychiatry, vol. 56, no. 4, pp. 286296, 2017.

[4] M. C. Mann-Wrobel, J. T. Carreno, and D. Dickinson, "Metaanalysis of neuropsychological functioning in euthymic bipolar disorder: an update and investigation of moderator variables," Bipolar Disorders, vol. 13, no. 4, pp. 334-342, 2011.

[5] S. K. Syan, M. Smith, B. N. Frey et al., "Resting-state functional connectivity in individuals with bipolar disorder during clinical remission: a systematic review," Journal of Psychiatry \& Neuroscience, vol. 43, no. 5, pp. 298-316, 2018.

[6] Y. Zhong, C. Wang, W. Gao et al., "Aberrant resting-state functional connectivity in the default mode network in pediatric bipolar disorder patients with and without psychotic symptoms," Neuroscience Bulletin, vol. 35, no. 4, pp. 581-590, 2019.

[7] K. K. Ellard, A. K. Gosai, J. M. Felicione et al., "Deficits in frontoparietal activation and anterior insula functional connectivity during regulation of cognitive-affective interference in bipolar disorder," Bipolar Disorders, vol. 21, no. 3, pp. 244258, 2019.

[8] M. N. Pavuluri, A. M. Passarotti, E. M. Harral, and J. A. Sweeney, "An fMRI study of the neural correlates of incidental versus directed emotion processing in pediatric bipolar disorder," Journal of the American Academy of Child and Adolescent Psychiatry, vol. 48, no. 3, pp. 308-319, 2009.

[9] M. R. Arbabshirani, M. Havlicek, K. A. Kiehl, G. D. Pearlson, and V. D. Calhoun, "Functional network connectivity during rest and task conditions: a comparative study," Human Brain Mapping, vol. 34, no. 11, pp. 2959-2971, 2013.

[10] S. M. Smith, P. T. Fox, K. L. Miller et al., "Correspondence of the brain's functional architecture during activation and rest," Proceedings of the National Academy of Sciences of the United States of America, vol. 106, no. 31, pp. 13040-13045, 2009.
[11] X. Chen, M. Duan, Q. Xie et al., "Functional disconnection between the visual cortex and the sensorimotor cortex suggests a potential mechanism for self-disorder in schizophrenia," Schizophrenia Research, vol. 166, no. 1-3, pp. 151-157, 2015.

[12] C. Luo, S. Tu, Y. Peng et al., "Long-term effects of musical training and functional plasticity in salience system," Neural Plasticity, vol. 2014, Article ID 180138, 13 pages, 2014.

[13] C. Vargas, C. Lopez-Jaramillo, and E. Vieta, "A systematic literature review of resting state network-functional MRI in bipolar disorder," Journal of Affective Disorders, vol. 150, no. 3, pp. 727-735, 2013.

[14] W. G. Cao, L. Chen, Q. C. Jiao, and L. H. Ding, "Spatial orientation interaction mechanism between chondroitin sulfate and azure A," Guang Pu Xue Yu Guang Pu Fen Xi, vol. 23, no. 3, pp. 587-590, 2003.

[15] R. L. Zhang, Q. Jiao, and B. G. Wang, "Controlled clinical study on 49 patients of SARS treated by integrative Chinese and Western medicine," Zhongguo Zhong Xi Yi Jie He Za Zhi, vol. 23, no. 9, pp. 654-657, 2003.

[16] L. Dong, C. Luo, X. Liu et al., "Neuroscience information toolbox: an open source toolbox for EEG-fMRI multimodal fusion analysis," Frontiers in Neuroinformatics, vol. 12, p. 56, 2018.

[17] D. Sridharan, D. J. Levitin, and V. Menon, "A critical role for the right fronto-insular cortex in switching between centralexecutive and default-mode networks," Proceedings of the National Academy of Sciences, vol. 105, no. 34, pp. 1256912574, 2008.

[18] Y. Jiang, M. Xia, X. Li et al., "Insular changes induced by electroconvulsive therapy response to symptom improvements in schizophrenia," Progress in Neuro-Psychopharmacology \& Biological Psychiatry, vol. 89, pp. 254-262, 2019.

[19] L. Palaniyappan and P. F. Liddle, "Does the salience network play a cardinal role in psychosis? An emerging hypothesis of insular dysfunction," Journal of Psychiatry \& Neuroscience, vol. 37, no. 1, pp. 17-27, 2012.

[20] S. A. Cardenas, L. Kassem, M. A. Brotman, E. Leibenluft, and F. J. McMahon, "Neurocognitive functioning in euthymic patients with bipolar disorder and unaffected relatives: a review of the literature," Neuroscience and Biobehavioral Reviews, vol. 69, pp. 193-215, 2016.

[21] D. Barry and N. M. Petry, "Predictors of decision-making on the Iowa Gambling Task: independent effects of lifetime history of substance use disorders and performance on the Trail Making Test," Brain and Cognition, vol. 66, no. 3, pp. 243252, 2008.

[22] W. Van der Elst, M. P. Van Boxtel, G. J. Van Breukelen, and J. Jolles, "Detecting the significance of changes in performance on the Stroop Color-Word Test, Rey's Verbal Learning Test, and the Letter Digit Substitution Test: the regression-based change approach," Journal of the International Neuropsychological Society, vol. 14, no. 1, pp. 71-80, 2008.

[23] V. Menon and L. Q. Uddin, "Saliency, switching, attention and control: a network model of insula function," Brain Structure \& Function, vol. 214, no. 5-6, pp. 655-667, 2010.

[24] C. F. Chang, T. Y. Hsu, P. Tseng et al., "Right temporoparietal junction and attentional reorienting," Human Brain Mapping, vol. 34, no. 4, pp. 869-877, 2013.

[25] J. Decety and C. Lamm, "The role of the right temporoparietal junction in social interaction: how low-level computational processes contribute to meta-cognition," The Neuroscientist, vol. 13, no. 6, pp. 580-593, 2007. 
[26] S. C. Krall, C. Rottschy, E. Oberwelland et al., "The role of the right temporoparietal junction in attention and social interaction as revealed by ALE meta-analysis," Brain Structure \& Function, vol. 220, no. 2, pp. 587-604, 2015.

[27] M. P. Lopez-Larson, L. M. Shah, H. R. Weeks et al., "Abnormal functional connectivity between default and salience networks in pediatric bipolar disorder," Biol Psychiatry Cogn Neurosci Neuroimaging, vol. 2, no. 1, pp. 85-93, 2017.

[28] C. Luo, Q. Li, Y. Lai et al., "Altered functional connectivity in default mode network in absence epilepsy: a resting-state fMRI study," Human Brain Mapping, vol. 32, no. 3, pp. 438-449, 2011.

[29] S. J. Broyd, C. Demanuele, S. Debener, S. K. Helps, C. J. James, and E. J. S. Sonuga-Barke, "Default-mode brain dysfunction in mental disorders: a systematic review," Neuroscience and Biobehavioral Reviews, vol. 33, no. 3, pp. 279-296, 2009.

[30] D. Vatansever, A. E. Manktelow, B. J. Sahakian, D. K. Menon, and E. A. Stamatakis, "Angular default mode network connectivity across working memory load," Human Brain Mapping, vol. 38, no. 1, pp. 41-52, 2017.

[31] P. D. Butler, S. M. Silverstein, and S. C. Dakin, "Visual perception and its impairment in schizophrenia," Biological Psychiatry, vol. 64, no. 1, pp. 40-47, 2008.

[32] L. A. Thomas, M. A. Brotman, B. L. Bones et al., "Neural circuitry of masked emotional face processing in youth with bipolar disorder, severe mood dysregulation, and healthy volunteers," Developmental Cognitive Neuroscience, vol. 8, pp. 110-120, 2014.

[33] W. L. Tseng, B. L. Bones, R. R. Kayser et al., "An fMRI study of emotional face encoding in youth at risk for bipolar disorder," European Psychiatry, vol. 30, no. 1, pp. 94-98, 2015.

[34] W. Gao, Q. Jiao, S. Lu et al., "Alterations of regional homogeneity in pediatric bipolar depression: a resting-state fMRI study," BMC Psychiatry, vol. 14, no. 1, p. 222, 2014.

[35] M. L. Phillips, M. J. Travis, A. Fagiolini, and D. J. Kupfer, "Medication effects in neuroimaging studies of bipolar disorder," The American Journal of Psychiatry, vol. 165, no. 3, pp. 313-320, 2008. 